\title{
Method for Detecting the Inside of Coke Drum Using Acoustic Signals
}

\author{
Qian Guo, ${ }^{1,2}$ Xinglin Tong, ${ }^{1}$ Cui Zhang, ${ }^{1}$ Chengwei Deng, ${ }^{1}$ Baolin Zhang, \\ Qiao Xiong, ${ }^{1}$ and Chaoran Zhou ${ }^{1}$ \\ ${ }^{1}$ National Engineering Laboratory for Fiber Optic Sensing Technology, Wuhan University of Technology, Wuhan, Hubei 430070, China \\ ${ }^{2}$ Air Force Early Warning Academy, Wuhan, Hubei 430019, China
}

Correspondence should be addressed to Xinglin Tong; tongxinglin@whut.edu.cn

Received 20 April 2017; Accepted 28 May 2017; Published 27 June 2017

Academic Editor: Jesus Corres

Copyright (C) 2017 Qian Guo et al. This is an open access article distributed under the Creative Commons Attribution License, which permits unrestricted use, distribution, and reproduction in any medium, provided the original work is properly cited.

A distance and acoustic intensity reverberation (DAIR) physical model is developed that can be successfully applied to the signal processing of the hydraulic decoking process online monitoring. In this model, the transmission characteristics of acoustic signals generated by a moving sound source in a dynamic confined space are first analyzed using data recursion and correction according to the coordinate continuity in adjacent area and adjacent time. The results show that the nondetection zone of acoustic signals generated directly by the impact of water is eliminated, and the surface distribution of coke in the drum can be mapped in real time.

\section{Introduction}

At present, several methods have been used for hydraulic decoking monitoring [1-4]. The traditional methods can accurately detect the status of the coke by detecting the unique frequency of the acoustic vibration signals generated by high pressure water impacting the drum wall during the process of removing coke [5, 6]. Furthermore, Wang et al. [7] found that the total energy spectrum distribution of vibration signals generated at the same height increased according to the fixed curve of the drum. Nevertheless, when the layer of coke is thick, the acoustic vibration signals generated higher than $400 \mathrm{~Hz}$ are completely absorbed by the coke layer when the high pressure water impacts the coke surface in the axis region of the coke drum.

Conversely, acoustic signals lower than $400 \mathrm{~Hz}$ can be detected by optical fiber Fabry-Perot (F-P) sensors, which changes intensity explicitly only in the early stage of the process. The optical fiber F-P sensors have a wide range of response frequency, which is especially sensitive to low frequency signals [8]. In addition, the optical fiber F-P sensors have the advantages of a simple structure, light weight, antielectromagnetic interference, corrosion resistance, high sensitivity, and high security and stability, which overcomes the limitations of mechanical and electrical sensors $[9,10]$.

According to the principle of reverberation generated in architectural acoustics [11], when the coke layer is thick, multiple reflection and scattering of the low frequency signals in the narrow space of the drum will cause a reverberation. The reverberation has a lower degree of absorption than the high frequency signals, which is sensitive to the F-P optical fiber sensor. The low frequency band-pass vibration signals could exist until the high frequency signals appear, and the relationship between the attenuation and the formation of the space expansion inside the drum has a certain regularity.

This paper presents a method for monitoring the state of hydraulic coke removal, which is divided into two stages. One stage is a conventional measurement; when the coke layer is thin, the high frequency sound vibration signals generated by the high pressure water jet impacting the coke or the drum wall surface have a mature method to be collected [12]. In this stage, the thickness of the coke layer can be analyzed using signal acquisition and frequency parameter measurement. Another stage is when the coke layer is thick during the early stage, or under abnormal conditions, such as coke collapse. The ratio of high frequency sound vibration signals is too 


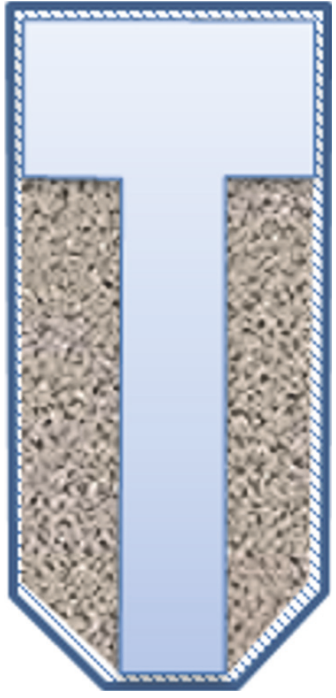

(a)

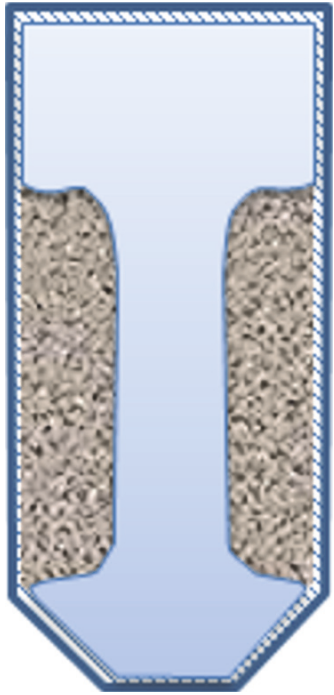

(b)

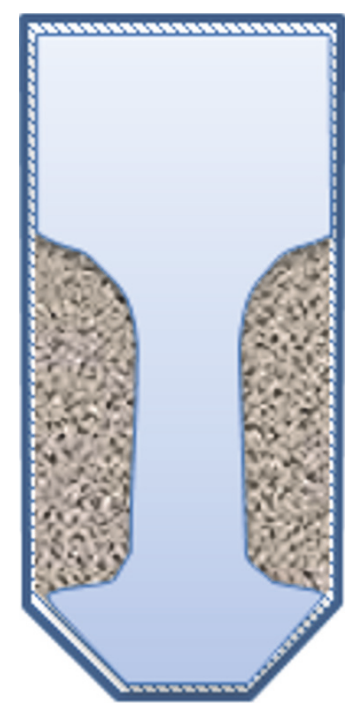

(c)

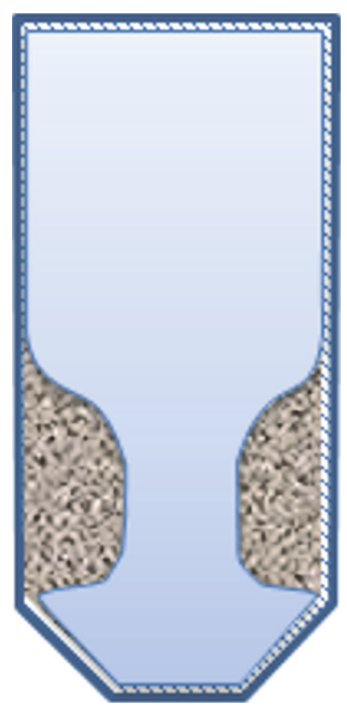

(d)

FIGURE 1: (a)-(d) Changes in internal space of the coke drum.

weak to be collected, while a lower frequency sound vibration signals can be monitored. Through spectral distribution analysis and the law of reverberation, the thickness variation of the coke layer could be estimated.

\section{Principles of Reverberation in Closed Coke Drum}

The sound detected by optical fiber F-P sensors is composed of both direct sound and reverberation sound. The direct sound is generated by the water jet impacting obstacles, which means that the position of the sound source varies with the rotation and vertical movement of the drill pipe. When acoustic waves projected into the irregular surface inside the coke drum, they will scatter and reflect.

Reverberation technology is mostly used to improve sound effects and for other commercial purposes [13-16]. Due to its scattering properties, it is rarely used in the field of measurement. According to the theory of reverberation, high frequency reverberation is easy attenuate, while the low frequency reverberation decay time is long, and the attenuation process is smooth $[17,18]$. In the early stage of the decoking, the contact point of the water jet on the coke layer is far away from the drum wall. The high frequency sound is absorbed by the coke and could not be detected by the sensors. Nevertheless, the coke formed a structure which could gather the acoustic signals well, as shown in Figures 1(a) and 1(b). After several times of signals reflection and scattering superposition, the high intensity of low frequency signals is measured by the F-P sensors. With the loss of coke, the carbon layer becomes thinner, and the structure changes gradually, as shown in Figures 1(c) and 1(d). The reflection efficiency of the coke pile shape becomes weakened, while the high frequency sound signals can be detected by the sensors to estimate the coke thickness linearly.
According to the distance and acoustic intensity reverberation (DAIR) physical model of sound propagation in closed space, the sound pressure level at $r$ from the sound source can be expressed as follows [17]:

$$
L_{p}=L_{w}+10 \lg \left(\frac{Q}{4 \pi r^{2}}+\frac{4}{R}\right) .
$$

Among them, $L_{w}$ in $\mathrm{dB}$ is the power level of the sound source. It is an approximate constant in the conditions of normal working modes with the constant pressure of the water jet. $r$ is the distance between the receiver and sound source. $Q$ is the directivity factor, which equals to 1 as the sound source is nondirectional. $R$ represents the sound absorption properties of the closed space, which is decided by the surface area of the space $S$ in $\mathrm{m}^{2}$ and the mean sound absorption coefficient $\bar{\alpha} . R$ can be expressed as follows:

$$
R=\frac{S \bar{\alpha}}{1-\bar{\alpha}} .
$$

There are two types of materials on the inner surface of the coke drum, coke and steel. Therefore, according to the definition of the mean sound absorption coefficient [17], $\bar{\alpha}$ can be expressed as follows:

$$
\bar{\alpha}=\frac{\alpha_{1} S_{1}+\alpha_{2} S_{2}}{S},
$$

where $S_{1}$ is the surface area of steel and $S_{2}$ is the surface area of coke. $\alpha_{1}$ and $\alpha_{2}$ are the sound absorption coefficients of steel and coke.

With hydraulic coke removal, the total area $S$ and coke area $S_{2}$ gradually reduced, while the area of steel $S_{1}$ gradually increased. Replacing $\left(\alpha_{1} S_{1}+\alpha_{2} S_{2}\right)$ with $\beta \cdot S$, the sound pressure level can be recursively calculated by (1) (3) as follows:

$$
L_{p}=L_{w}+10 \lg \left[\frac{1}{4 \pi r^{2}}+\frac{4}{S}\left(\frac{1}{\beta}-1\right)\right] .
$$




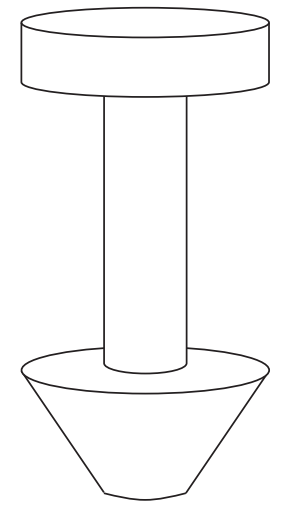

(a)

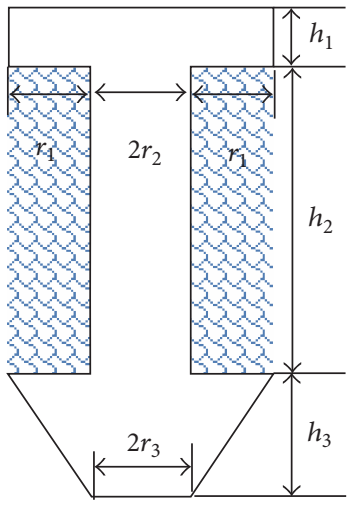

(b)

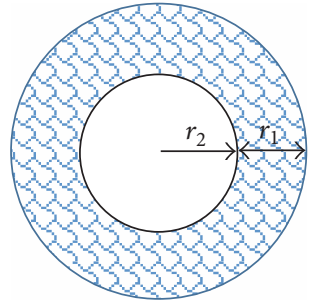

(c)

FIGURE 2: (a) The 3D structure diagram of the geometric model of internal spatial structure; (b) the longitudinal section of the geometric model; (c) the cross section of the geometric model.

From (4), it can be seen that the measured reverberation should be inversely proportional to the square of $r$ and the sum surfaces are $S$. It is almost impossible to obtain a model that is fully consistent with reality because of the randomness of coke formation. Therefore, we developed a simplified approximate geometric model to obtain $S_{1}$ and $S_{2}$, as shown in Figure 2.

In the process of coke removal, the simplified geometric model of the internal spatial structure is divided into three parts in a vertical direction, with their respective heights denoted by $h_{1}, h_{2}$, and $h_{3} . h_{1}$ represents the height of the coke removal region. The initial value of $h_{1}$ is measured by a radioactive level meter on the coke drum, which is provided at the beginning of the decoking operation. Along with the decoking, $h_{1}$ can be confirmed by the characterization of high intensity signals generated by the high pressure water jet hitting the metal drum wall detected throughout the region. $h_{3}$ is a constant value equal to the height of the cone on the bottom of the drum. $h_{2}$ can be derived using $h_{1}$ and $h_{3}$ and the height of the drum $h_{0}$ such that $h_{2}=h_{0}-h_{1}-h_{3}$. The variable $r_{2}$ is radius of the voids formed by residual coke, and $r_{1}$ is the thickness of coke. In Figure 2(b), the blue region expresses the coke region. Therefore, the junction of the blue patterned area and the geometric model is approximated as the surface of the coke, whose area can be expressed as follows:

$$
S_{2}=2 \pi \int_{0}^{h_{2}} r_{2}(h) d h .
$$

The surface area of steel is

$$
\begin{aligned}
S_{1}= & \pi r_{0}^{2}+2 \pi r_{0} h_{1}+\pi r_{3}^{2} \\
& +\pi\left(r_{0}+r_{3}\right) \sqrt{h_{3}^{2}+\left(r_{0}-r_{3}\right)^{2}},
\end{aligned}
$$

where $r_{0}$ represents the radius of the coke drum and $r_{3}$ is the inner bottom radius of coke drum.

The distance between the sensor and the source $r$ can be expressed as follows:

$$
r^{2}=r_{0}^{2}+r_{2}^{2}-2 r_{0} r_{2} \cos \left(\varphi_{0}+\omega t\right)+|\Delta h(t)|^{2},
$$

where $\omega$ is the rotation speed of the drill pipe, $\varphi_{0}$ is the initial phase of the drill pipe, $t$ is the time of the rotation, and $\Delta h(t)$ is the height difference of the sensor and the action point of water which can be determined by the height of the drill.

In real-time monitoring, the current reverberation intensity is determined by internal surface area of the coke drum at a previous time. Therefore, we use the last instantaneous $S_{1}$ and $S_{2}$ as references to calculate the present $r$ by (4). As an analogy, $r$ can be deduced at any time and horizontal direction. Additionally, the new thickness at the same direction and height should replace the old one, and the distribution of the coke surface in the coke drum can be depicted using all of the newest thicknesses.

We can conclude that the coke thickness can be determined at any height by measuring the intensity of low frequency acoustic vibration signals generated by reverberation, which is combined with the DAIR model presented above.

\section{Experiment}

The data used for the experimental analysis is provided by a monitoring system, as shown in Figure 3. The monitoring system consists of a group of F-P sensors, an optical fiber sensor demodulator, and an industrial personal computer.

The F-P sensor group contains five acoustic vibration F-P sensors, each of which converts the sound intensity of the mounting point to the feedback intensity with the same structure and performance described in [12]. The F-P sensor manufactured by our laboratory can sense the sound source from a distance of more than ten meters. Thus the measured range of the $5 \mathrm{~F}-\mathrm{P}$ sensors vertical distribution can cover the entire 35.2-meter coke drum.

The optical fiber sensor demodulator system integrates a laser light source, optical splitter, optical circulator, opticalelectrical conversion circuit, and signal acquisition card. It provides a wavelength tunable light source for the sensors, which ensures the sensors are maintained in linear demodulation range at various temperatures and conditions. Another function is the optical signal conversion, the electronic signal acquisition, and transmission to the host computer. The 


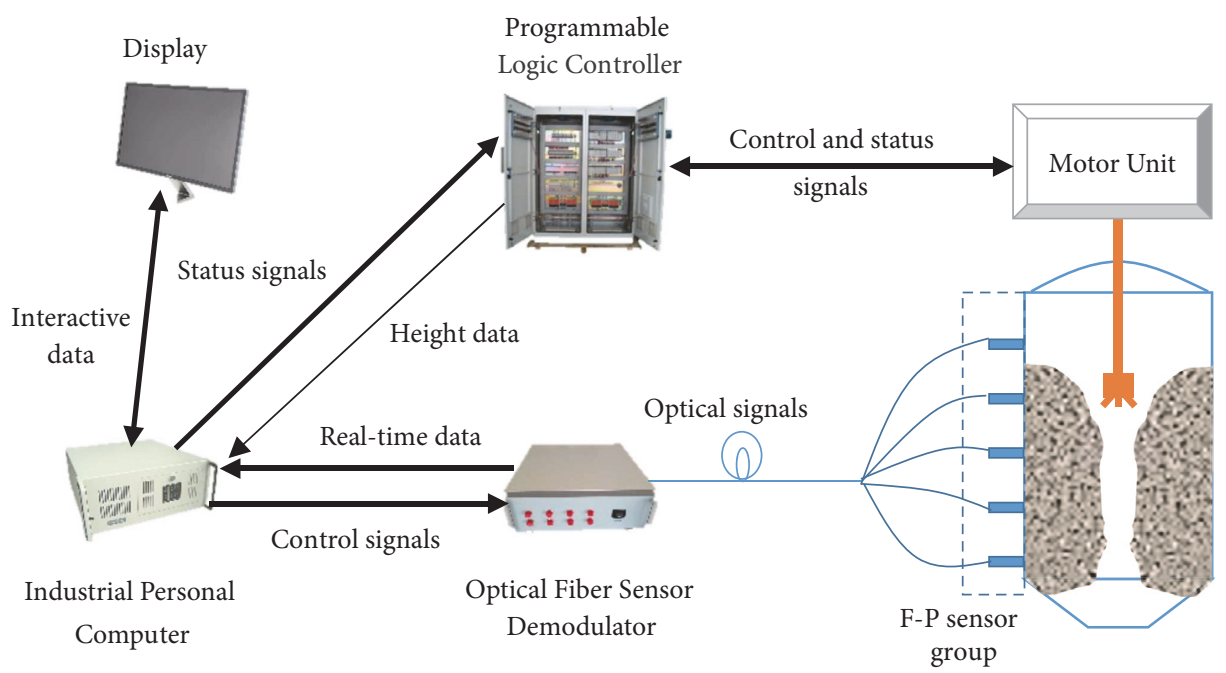

Figure 3: The schematic diagram of data acquisition based on the optical fiber sensor monitoring system.

parameters settings and operative modes are controlled by the host computer.

During the coke cutting stage, the position of the drill and the position of the high pressure water impacting the coke or the wall are approximately equal in height. The industrial computer is used as the host computer to read the height signal of the drill bit from the coke removal control system in real time. According to the height value, the sampling data of the nearest sensor is selected for analysis and storage. The coke removal control system is the original equipment of Wuhan Petrochemical Company.

\section{Results and Discussions}

Figure 4 shows the waveform of the signals acquired during the process of hydraulic coke removal from height of 19 meters to 23.7 meters, with a sampling frequency of $10 \mathrm{kHz}$. The horizontal axis represents the cutting operating time of the region, which is incompletely continuous in the whole process depending on the operation steps. In the process of mechanical operation, a short time strong interference noise is unavoidable, which could be filtered out in further analysis.

It can be seen that the average amplitude of the signals has an upward trend, as shown in Figure 4(a). What should not be ignored is that the average amplitude of early signals is relatively low and stable, or even following a downward trend in Figure 4(b). This suggests that the signals obtained are not only the sound source generated directly, but also the reverberation formed in the confined space.

The method of frequency analysis in this paper is based on fast Fourier transform (FFT) with gliding window. The width of the gliding window is $1 \mathrm{~s}$.

The characteristic curve is obtained using a timefrequency analysis after filtering the background noise, as shown in Figure 5(a). And Figure 5(b) shows the variety of the total energy of signals during the entire process. We can observe from the time-frequency characteristics that the energy of signals below $400 \mathrm{~Hz}$ occupies a large proportion of

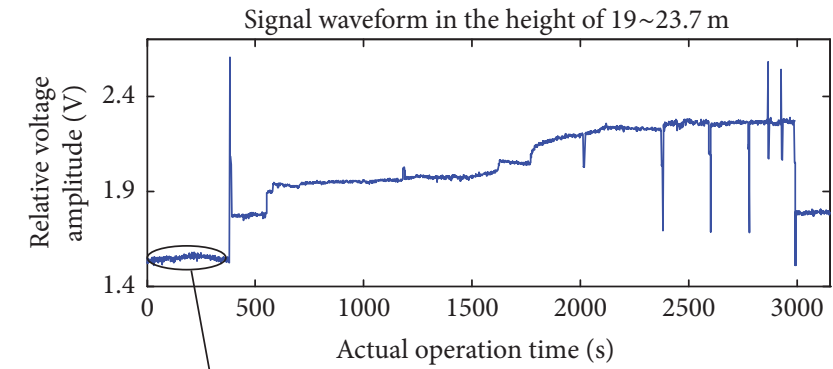

(a)

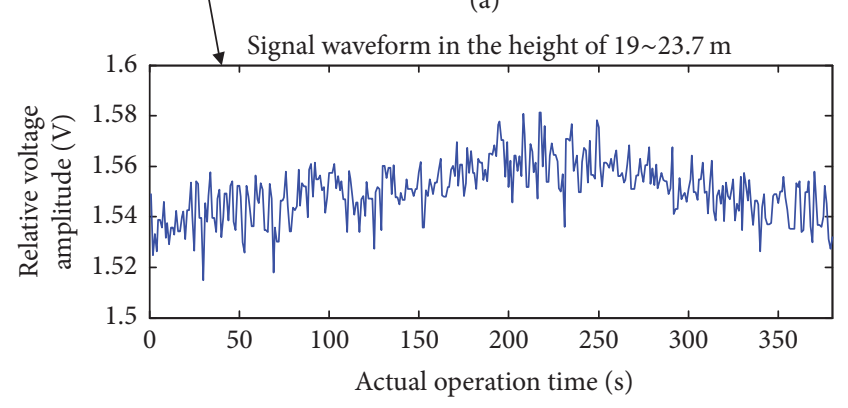

(b)

FIgURE 4: (a) The waveform of the signals acquired in the whole process (for $3150 \mathrm{~s}$ ) of a hydraulic coke removal at the height of 19 meters to 23.7 meters; (b) enlarged detail of the waveform of the signals in the early stage (for $380 \mathrm{~s}$ ).

the total energy, especially in the early stage of the operation. In the process of coke cutting, the energy is dispersed into higher frequency band, which is the reason for total energy growth despite no obvious increase of low frequency signals.

In order to conveniently observe and analysis data, signals below $400 \mathrm{~Hz}$ are extracted independently. Figure 6(a) shows the energy distribution from 0 to $400 \mathrm{~Hz}$. Through the peak searching, several frequency points with energy concentrated are analyzed, as shown in Figures 6(b)-6(g). From the results, we indicate that the energy of reverberation is mainly 


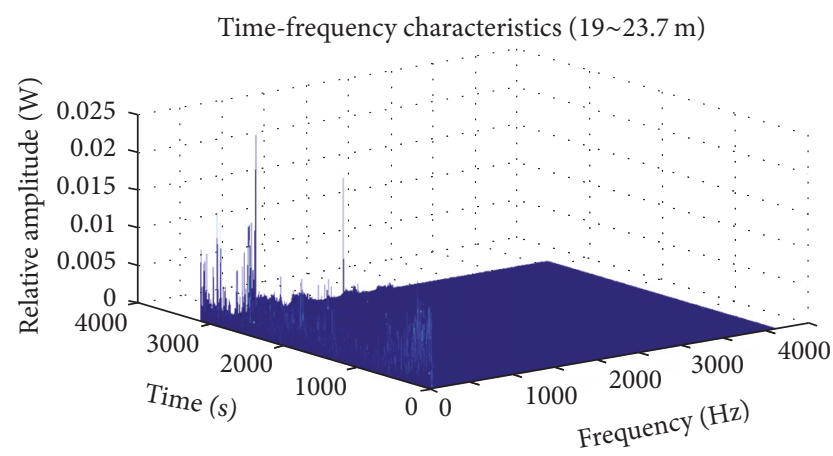

(a)

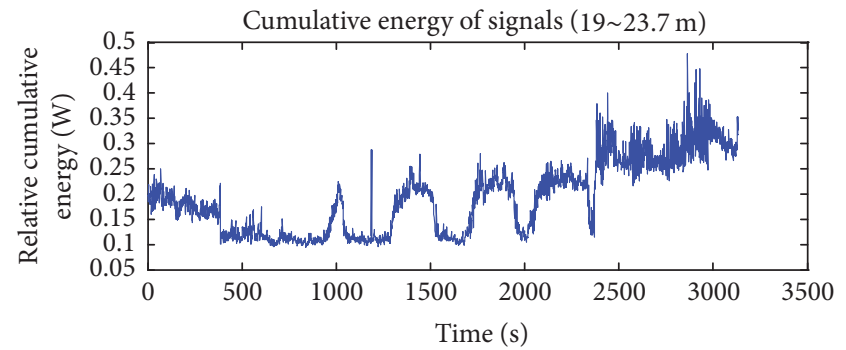

(b)

Figure 5: (a) The time-frequency characteristics of the signals at the height of 19 meters to 23.7 meters; (b) the variety of total energy of signals in the whole process.

concentrated in the frequency bands from $9 \mathrm{~Hz}$ to $288 \mathrm{~Hz}$. It can be seen from the energy variety of each frequency point that the energy is decreased gradually until stable. Furthermore, the details of the energy change curve of single frequency point are similar to the cosine trend, whose cycle is in accord with the rotation speed of the drill pipe. All the results demonstrate that the DAIR model of reverberation proposed is consistent with the actual situation.

Figure 7 shows the cumulative energy curve of signals from 9 to $124 \mathrm{~Hz}$ in the same height region as the above analysis. According to the DAIR model, the results of the relative energy can express the intensity ratio of the signals detected to the sound source signal linear, with a fixed coefficient determined by the experimental system.

The surface area of the coke can be calculated with the average radius of the hole formed by coke cutting at each height. Thus, the industrial computer needs to record each operation valuation to be called for the model, whose initial values are set to 0 . In order to reduce computation, 5 points on each height are recorded, which means the horizontal angular resolution is $72^{\circ}$.

According to the DAIR model, we calculate the location of each instantaneous sound source, and depict them in the three-dimensional space map, as shown in Figure 8. The end of the blue lines indicates the location of the sound source from $4^{\prime} 51$ to $9^{\prime} 51 \mathrm{~s}$, while the red circles represent from $24^{\prime} 50$ to $26^{\prime} 10 \mathrm{~s}$. The location of the sound source is the point where the coke is impacted by water. Therefore, the space formed by all the points recorded is the hole formed by the coke cutting. As can be seen from Figure 8, in time, the hole becomes larger. The resolution on the vertical height is affected by the high signal accuracy provided by the equipment, which can reach $0.1 \mathrm{~m}$.

The above method is applied to calculate the thicknesses of coke by the feature parameters of reverberation signals, when the signals have not attenuated too weakly to be distinguished. There is an intermediate process of coke removal, in which high frequency signals can be detected, and the enhancement of reverberation to low frequency signals still works. The amplitude variation of the higher frequency signals in this process was proved to be linear to the thickness variation of the coke layer [12]. The thicknesses of the coke calculated by the higher frequency signal characteristics are compared with the thicknesses calculated by the reverberation model in this paper, as shown in Table 1 .

Table 1 lists the differences $\Delta$ of the average coke thickness values of the same height layer obtained between the two methods during the decoking process. It selects the period of $28^{\prime} 10$ to $28^{\prime} 21$, and $55^{\prime} 38$ to $55^{\prime} 49$, in which the higher frequency signals and reverberation signals can be measured at the same time. It can be seen that the thicknesses calculated by the two measurements differ in centimeter range. Therefore, the thickness calculated using the DAIR model completely achieves the precision request of decoking monitoring.

\section{Conclusion}

This paper proposes an improved DAIR model based on reverberation, which uses the relationship between the transmission distance of the moving sound source and the reverberation intensity, to estimate the coke surface morphology in the coke drum. It provides a new method for monitoring the process of hydraulic coke removal and overcomes the issue of a blind area detected in other methods. Compared with traditional sensor detection methods, the entire process of hydraulic coke removal is monitored, which can provide early warnings of abnormal condition. The experimental results illustrate that the inner surface of the coke drum can be described according to the data detected in real time of hydraulic coke removal process, with the vertical accuracy of $0.1 \mathrm{~m}$ and the level of angular resolution of $72^{\circ}$.

\section{Conflicts of Interest}

The authors declare that they have no conflicts of interest.

\section{Acknowledgments}

This research was supported by the National High Technology Research and Development Program of China [863 Project, Grant no. 2015AA043505], and the National Nature 


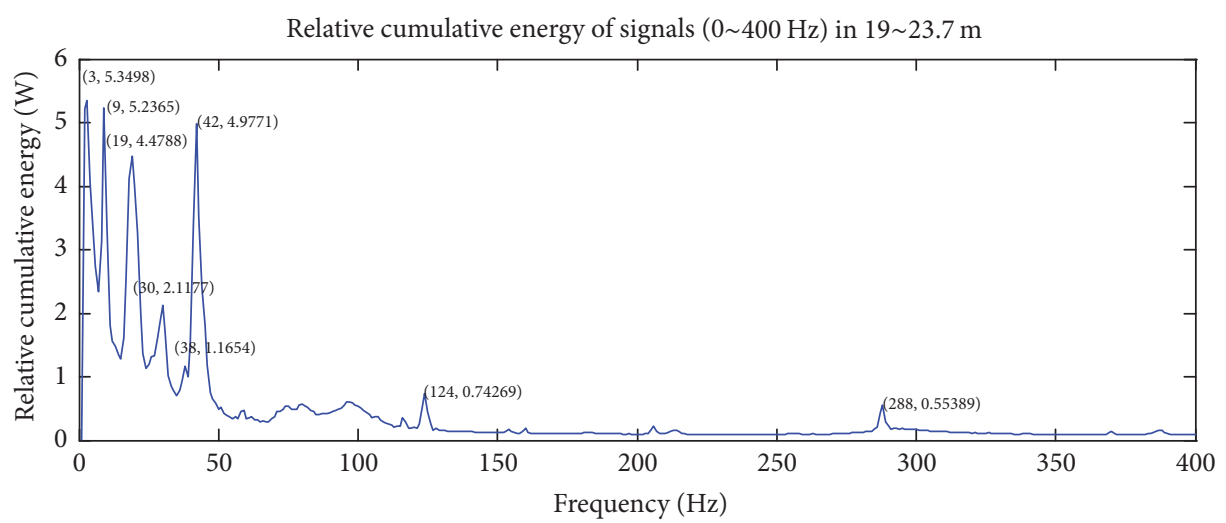

(a)

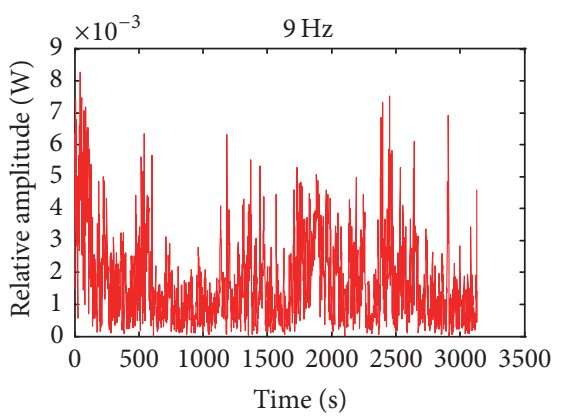

(b)

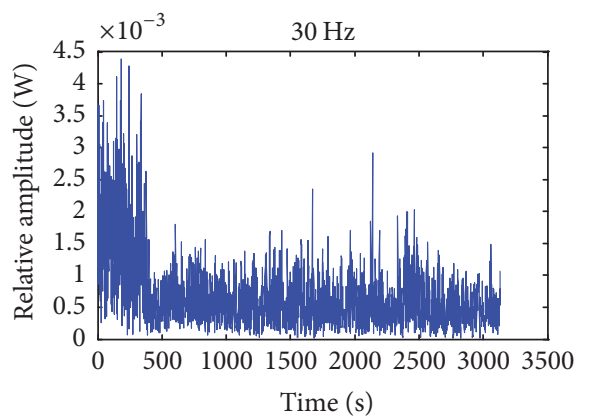

(d)

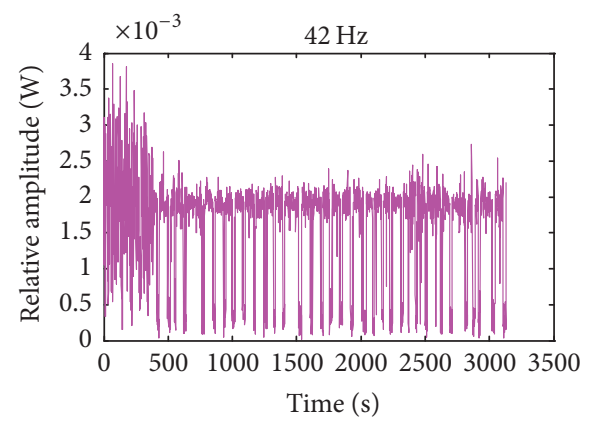

(f)

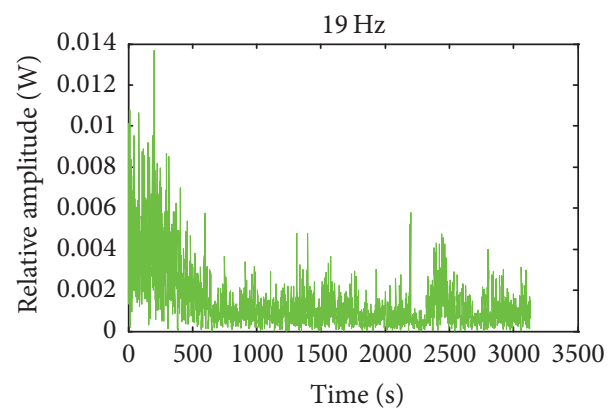

(c)

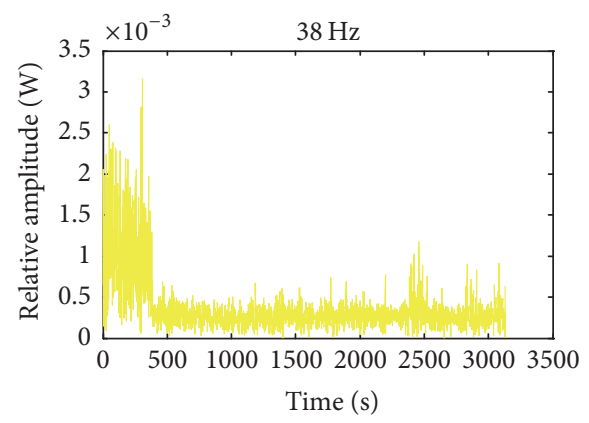

(e)

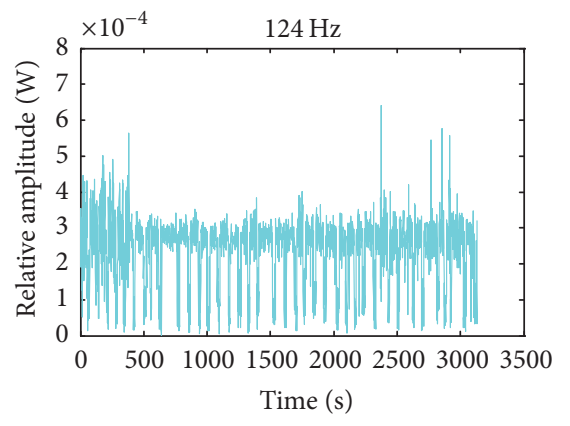

(g)

Figure 6: (a) The energy spectrum of the signals at a height of 19 meters to 23.7 meters; (b)-(g) the energy variety of key frequency at 9 Hz, $19 \mathrm{~Hz}, 30 \mathrm{~Hz}, 38 \mathrm{~Hz}, 42 \mathrm{~Hz}$, and $124 \mathrm{~Hz}$. 
TABLE 1: Comparison of the average thicknesses of the coke obtained via the higher frequency signal characteristics (HFSC) and the DAIR model at the same times and heights.

\begin{tabular}{|c|c|c|c|c|c|}
\hline & $\begin{array}{c}19.3 \mathrm{~m} \\
28^{\prime} 21 \\
\end{array}$ & $\begin{array}{c}19.4 \mathrm{~m} \\
28^{\prime} 19 \\
\end{array}$ & $\begin{array}{c}19.5 \mathrm{~m} \\
28^{\prime} 15 \\
\end{array}$ & $\begin{array}{c}19.6 \mathrm{~m} \\
28^{\prime} 13 \\
\end{array}$ & $\begin{array}{c}19.7 \mathrm{~m} \\
28^{\prime} 10 \\
\end{array}$ \\
\hline HFSC & $327.800 \mathrm{~cm}$ & $332.353 \mathrm{~cm}$ & $324.917 \mathrm{~cm}$ & $331.663 \mathrm{~cm}$ & $328.868 \mathrm{~cm}$ \\
\hline DAIR & $328.385 \mathrm{~cm}$ & $331.768 \mathrm{~cm}$ & $324.133 \mathrm{~cm}$ & $330.261 \mathrm{~cm}$ & $328.262 \mathrm{~cm}$ \\
\hline \multirow[t]{2}{*}{$\Delta$} & $0.585 \mathrm{~cm}$ & $0.585 \mathrm{~cm}$ & $0.784 \mathrm{~cm}$ & $1.402 \mathrm{~cm}$ & $0.606 \mathrm{~cm}$ \\
\hline & $\begin{array}{c}25.1 \mathrm{~m} \\
55^{\prime} 49\end{array}$ & $\begin{array}{c}25.2 \mathrm{~m} \\
55^{\prime} 47\end{array}$ & $\begin{array}{c}25.3 \mathrm{~m} \\
55^{\prime} 43\end{array}$ & $\begin{array}{c}25.4 \mathrm{~m} \\
55^{\prime} 40\end{array}$ & $\begin{array}{c}25.5 \mathrm{~m} \\
55^{\prime} 38\end{array}$ \\
\hline HFSC & $284.413 \mathrm{~cm}$ & $285.482 \mathrm{~cm}$ & $283.710 \mathrm{~cm}$ & $281.258 \mathrm{~cm}$ & $285.868 \mathrm{~cm}$ \\
\hline DAIR & $283.011 \mathrm{~cm}$ & $283.621 \mathrm{~cm}$ & $282.367 \mathrm{~cm}$ & $281.961 \mathrm{~cm}$ & $286.572 \mathrm{~cm}$ \\
\hline$\Delta$ & $1.402 \mathrm{~cm}$ & $1.861 \mathrm{~cm}$ & $1.343 \mathrm{~cm}$ & $0.703 \mathrm{~cm}$ & $0.704 \mathrm{~cm}$ \\
\hline
\end{tabular}

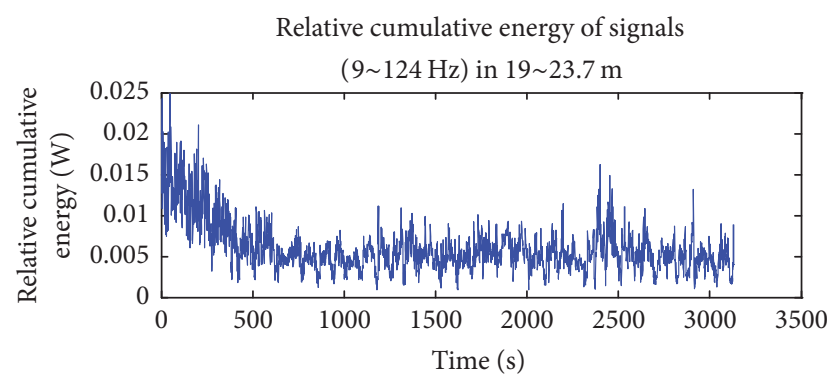

FIGURE 7: The cumulative energy curve of signals from 9 to $124 \mathrm{~Hz}$ at height of 19 23.7 m.

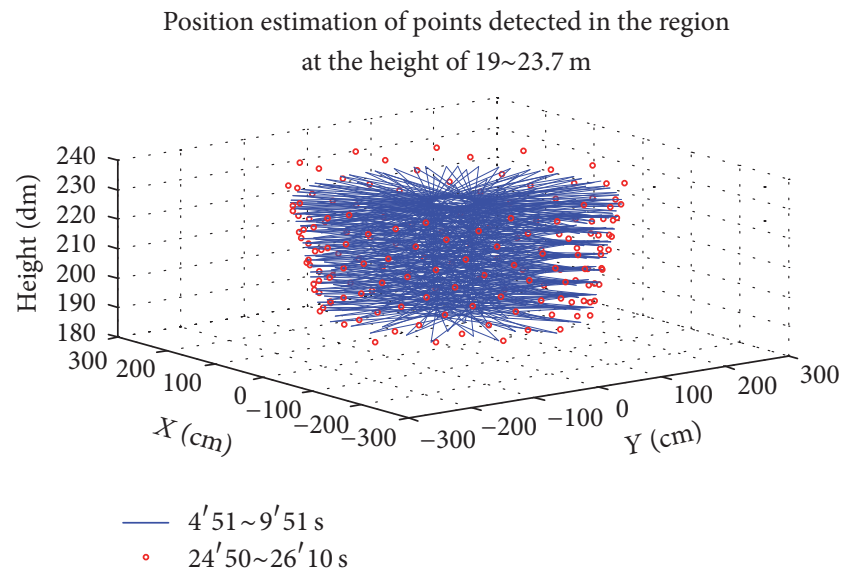

FIGURE 8: The position estimation of points at the height of 19 meters to 23.7 meters in different time segment.

Science Foundation of China [Grant no. 51275373 and Grant no. 61575148], and the Key Project of the Nature Science Foundation of Hubei provincial government [Grant no. 2014CFA056]. The authors would like to thank postgraduates Di Huang, Liang Chen, Minli Zhao, and others for their sound advice on the experiment.

\section{References}

[1] A. N. Sawarkar, A. B. Pandit, S. D. Samant, and J. B. Joshi, "Petroleum residue upgrading via delayed coking: a review," Canadian Journal of Chemical Engineering, vol. 85, no. 1, pp. 124, 2007.

[2] I. Botros, "Automated decoking solves coker safety challenges," Hydrocarbon Processing, vol. 90, no. 11, pp. 47-50, 2011.

[3] J. Lee and D.-H. Kim, "Experimental modal analysis and vibration monitoring of cutting tool support structure," International Journal of Mechanical Sciences, vol. 37, no. 11, pp. 1133-1146, 1995.

[4] X. Wang, "Pass balancing switching control of a four-passes furnace system," in Proceedings of the 18th IFAC World Congress, vol. 18, pp. 12078-12083, Hydrocarbon Processing, Milano, Italy, September 2011.

[5] Y.-F. Fan, X.-L. Tong, T. Ji, X.-Q. Gao, and D. Zhong, "Application of optical fiber sensing technology in the hydraulic decoking monitoring system," in Proceedings of the 4th AsiaPacific Optical Sensors Conference 2013, APOS 2013, vol. 8924, Wuhan, China, October 2013.

[6] H. A. Alkhazali and M. R. Askari, "Computer simulation to investigate system identification and vibration monitoring in rotor dynamic analysis," Chemical Reviews, vol. 107, no. 12, pp. 5813-5840, 2011.

[7] T. Wang, Z. Gao, J. Ye, and X. Hu, "Design and research on hydraulic coke cutting monitoring system," Journal of Engineering Design, vol. 17, no. 2, pp. 146-150, 2010.

[8] H. Yang, J. Jiang, S. Wang, Y. Pan, X. Zhang, and T. Liu, "Temperature-insensitive optical Fabry-Perot flow measurement system with partial bend angle," Chinese Optics Letters, vol. 15, no. 4, article 040601, 2017.

[9] J. Xu, X. Wang, K. L. Cooper, and A. Wang, "Miniature all-silica fiber optic pressure and acoustic sensors," Optics Letters, vol. 30, no. 24, pp. 3269-3271, 2005.

[10] Q. Wang and Q. Yu, "Polymer diaphragm based sensitive fiber optic Fabry-Perot acoustic sensor," Chinese Optics Letters, vol. 8, no. 3, pp. 266-269, 2010.

[11] F. A. Everest and K. C. Pohlmann, Master Handbook of Acoustics, McGraw-Hill Education, New York, NY, USA, 5th edition, 2001.

[12] P. Hu, X. Tong, M. Zhao et al., "Study on high temperature Fabry-Perot fiber acoustic sensor with temperature selfcompensation," Optical Engineering, vol. 54, no. 9, Article ID 097104, 2015. 
[13] M. Meissner, "Acoustics of small rectangular rooms: analytical and numerical determination of reverberation parameters," Applied Acoustics, vol. 120, pp. 111-119, 2017.

[14] Y. Hioka, J. W. Tang, and J. Wan, "Effect of adding artificial reverberation to speech-like masking sound," Applied Acoustics, vol. 114, pp. 171-178, 2016.

[15] A. Prato, F. Casassa, and A. Schiavi, "Reverberation time measurements in non-diffuse acoustic field by the modal reverberation time," Applied Acoustics, vol. 110, pp. 160-169, 2016.

[16] M. L. Mehta, J. Johnson, and J. Rocafort, Architectural Acoustics: Principles and Design, Prentice Hall, Upper Saddle River, NJ, USA, 1999.

[17] Y. Jing and N. Xiang, "Visualizations of sound energy across coupled rooms using a diffusion equation model," Journal of the Acoustical Society of America, vol. 124, no. 6, pp. EL360-EL365, 2009.

[18] W. Kochański, M. Boeff, Z. Hashemiyan, W. J. Staszewski, and P. K. Verma, "Modelling and numerical simulations of in-air reverberation images for fault detection in medical ultrasonic transducers: a feasibility study," Journal of Sensors, vol. 2015, Article ID 796439, 14 pages, 2015. 


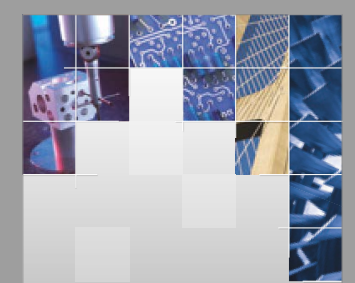

\section{Enfincering}
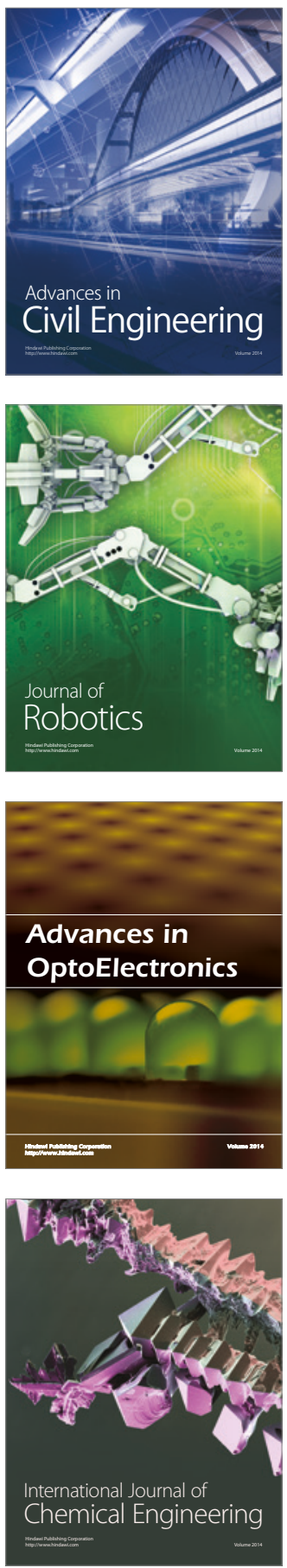

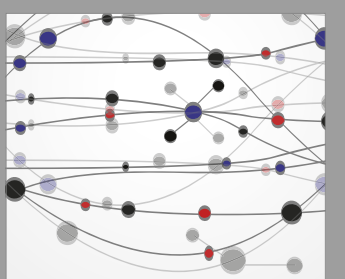

The Scientific World Journal

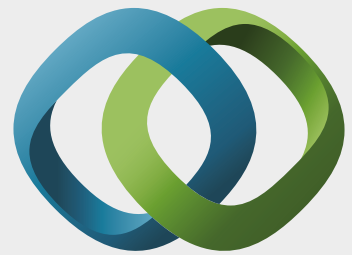

\section{Hindawi}

Submit your manuscripts at

https://www.hindawi.com
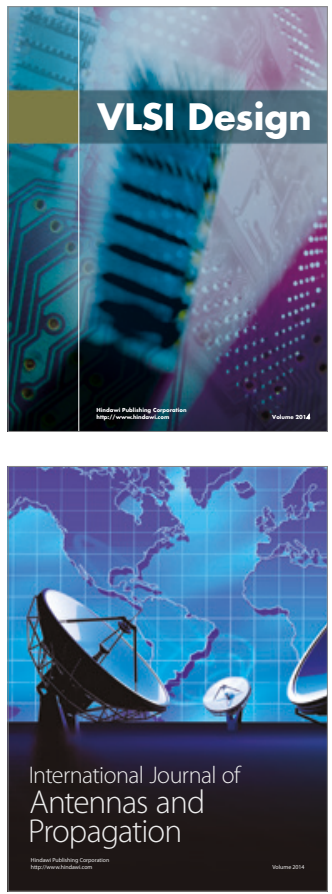

\section{Rotating}

Machinery
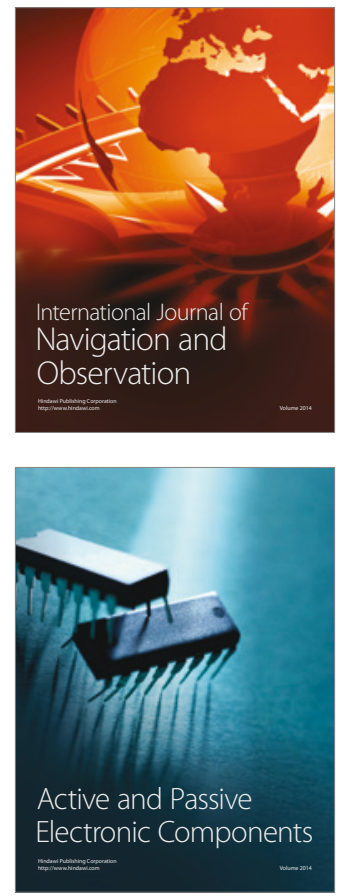
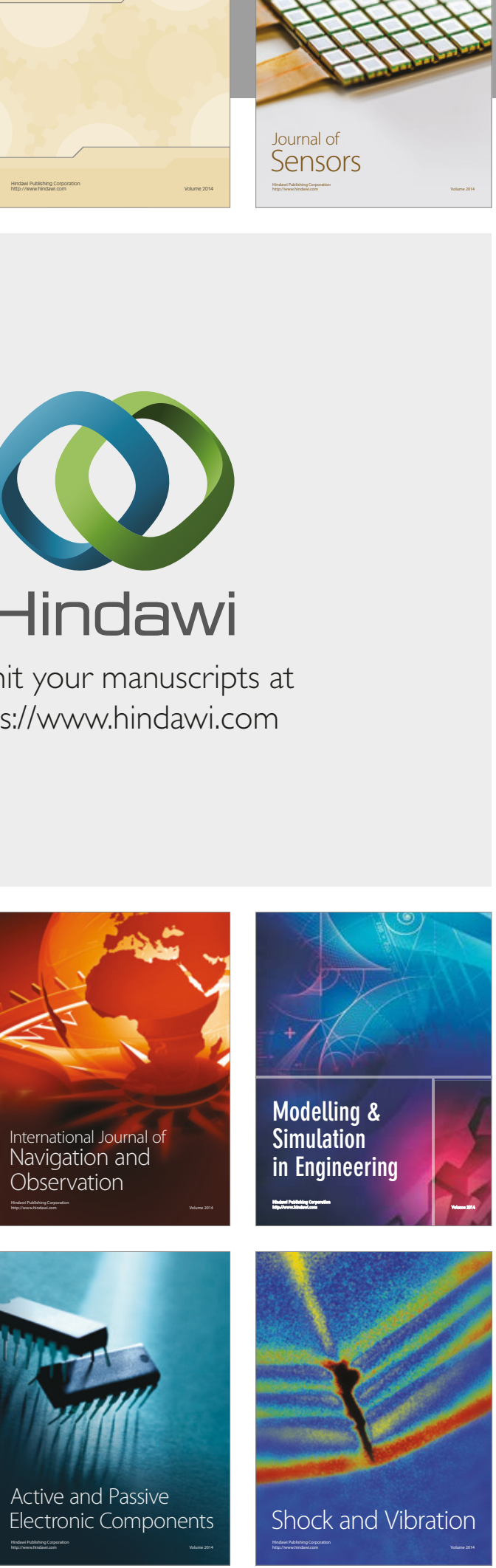
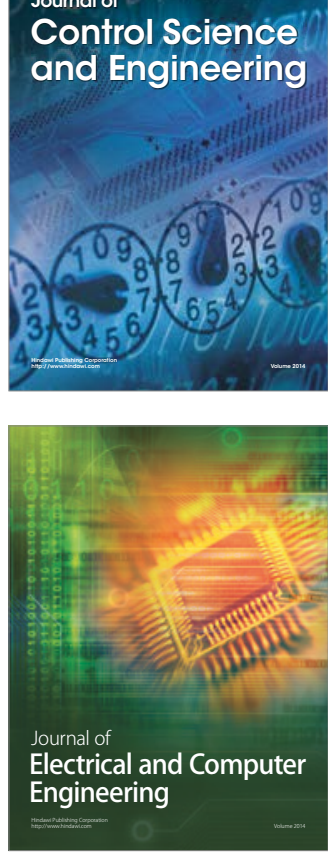

Distributed

Journal of

Control Science

and Engineering
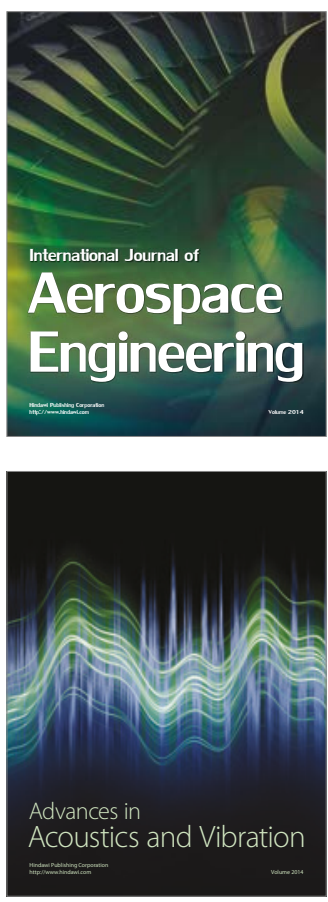

Sensor Networks 